\title{
Author Correction: Infectivity, susceptibility, and risk factors associated with SARS-CoV-2 transmission under intensive contact tracing in Hunan, China
}

Shixiong Hu, Wei Wang, Yan Wang, Maria Litvinova (D, Kaiwei Luo, Lingshuang Ren, Qianlai Sun, Xinghui Chen, Ge Zeng, Jing Li, Lu Liang, Zhihong Deng, Wen Zheng, Mei Li, Hao Yang, Jinxin Guo, Kai Wang, Xinhua Chen, Ziyan Liu, Han Yan, Huilin Shi, Zhiyuan Chen, Yonghong Zhou, Kaiyuan Sun, Alessandro Vespignani, Cécile Viboud, Lidong Gao, Marco Ajelli (i) \& Hongjie Yu (D)

Correction to: Nature Communications https://doi.org/10.1038/s41467-021-21710-6, published online 9 March 2021.

The original version of this Article contained an error in the first sentence of the introduction, which incorrectly read 'The outbreak of coronavirus disease 2019 (COVID-19) was first detected in December 2009 in Wuhan, China'. The first sentence of the introduction has been corrected to 'The outbreak of coronavirus disease 2019 (COVID-19) was first detected in December 2019 in Wuhan, China' in both the PDF and HTML versions of the Article.

Published online: 08 April 2021

\footnotetext{
(c) (i) Open Access This article is licensed under a Creative Commons Attribution 4.0 International License, which permits use, sharing, adaptation, distribution and reproduction in any medium or format, as long as you give appropriate credit to the original author(s) and the source, provide a link to the Creative Commons license, and indicate if changes were made. The images or other third party material in this article are included in the article's Creative Commons license, unless indicated otherwise in a credit

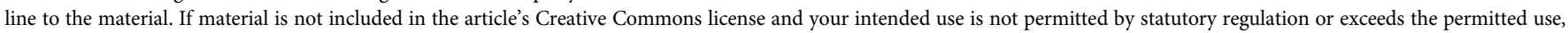
you will need to obtain permission directly from the copyright holder. To view a copy of this license, visit http://creativecommons.org/licenses/by/4.0/.
}

(C) The Author(s) 2021 